\title{
Nature et publicité sociale: du scénario catastrophe à la reconquête de l'environnement dans les campagnes de FNE
}

\section{Nature and advertising: from the catastrophic argument to the environment recovery in FNE campaigns}

\author{
MARINE ABRAHAM \\ Universidad de Murcia \\ marine.abraham1@um.es
}

\begin{abstract}
The aim of this study is to define the semiotic and linguistic components of social advertising, the objective of which is to raise awareness in western populations. To this end, we compare the procedures used in social and commercial posters, and highlight the importance of environmental issues in recent years, in which defending nature or commending natural concerns are more and more commonplace. Using a contrastive analysis of two campaigns by a French charitable organisation, France Nature Environnement (FNE), in 2011 and 2016, we reflect on the specific discursive strategies employed to seduce message receivers and demonstrate that both campaigns, the first through the use of irony and the second though the auguring of a better world, convince people of the need for sustainable development.
\end{abstract}

Key-words

social advertising, environment, linguistic, semiotic, irony.

\section{Resumen}

En este trabajo, determinamos los parámetros semio-lingüísticos de las publicidades sociales que tienen como objetivo la concienciación de las poblaciones occidentales en la actualidad. Para ello, comparamos los procedimientos utilizados en los carteles sociales y comerciales, y ponemos de relieve la importancia de la cuestión medioambiental en estos últimos años, razón por la cual los objetos publicitarios defendiendo la naturaleza o preconizando lo natural resultan ser cada vez más frecuentes. En concreto, a partir del análisis contrastivo de dos campañas de la asociación francesa France Nature Environnement (FNE) de 2011 y 2016, revelamos pues las estrategias discursivas concretas empleadas para seducir a los receptores de nuestras sociedades contemporáneas y evidenciamos que tanto la campaña de 2011 basada en la ironía como la de 2016 presagiando un mundo mejor consiguen persuadir a los individuos de la importancia de un planeta sostenible.

\section{Palabras clave}

publicidad social, medioambiente, lingüística, semiótica, ironía. 


\section{Introduction}

Produit culturel omniprésent dans le monde occidental, la publicité est à l'origine de nombreuses études tant sur les plans sémio-linguistique que psychologique, sociologique, culturel ou mercatique, entre autres. Son impact dans nos sociétés est tel que divers styles publicitaires se développent au $20^{\text {ème }}$ siècle, dont la publicité dite sociétale ou sociale qui deviendra un phénomène courant dès les années 1980. Incité par une ferveur croissante des populations envers la protection de l'environnement et leur désir de consommer de manière responsable, le poids des publicités sociales favorisant la défense du développement durable est de plus en plus considérable, raison pour laquelle leur analyse nous semble pertinente.

Ainsi, nous nous interrogerons sur les éléments socio-linguistiques composant certaines affiches sociétales actuelles et déterminerons de quelle manière les individus se voient séduits par ces objets publicitaires contemporains. Pour ce faire, nous nous intéresserons tout particulièrement aux deux campagnes menées par l'association France Nature Environnement (FNE) en 2011 et 2016. Il est question de deux séries d'affiches dont l'étude nous a paru opportune compte tenu des apports linguistiques et sémiotiques distinctifs de chaque campagne mais surtout en raison des deux types de discours employés qui se révèlent être caractéristiques des publicités sociales, le discours choc qui commotionne les récepteurs d'une part et le discours rassurant qui les réconforte d'autre part.

Dans un premier temps, nous allons comparer de manière générale les campagnes sociales et commerciales afin d'évaluer ce qui les rapproche et ce qui les éloigne. Puis nous évoquerons brièvement le poids de la nature dans les publicités du $21^{\text {ème }}$ siècle pour finalement nous centrer sur l'objet de notre étude, à savoir les affiches de l'organisme FNE. Puis, après avoir réalisé une analyse contrastive, tant au niveau linguistique que sémiotique, de ces deux campagnes, nous mènerons à bien une enquête de qualité par le biais d'un sondage afin de connaître quelle, des deux stratégies discursives proposées, est la plus à même de convaincre les individus.

\section{Cadre théorique}

\subsection{Publicités sociales et commerciales: similitudes et divergences}

L'histoire fait naître la publicité telle que nous la connaissons aujourd'hui aux ÉtatsUnis dans les années 1950, allant de pair avec le boom de la production durant les Trente Glorieuses qui entraînera plus tard celui des ventes et la démocratisation de certains produits et services. En revanche, la publicité sociale n'a commencé à se développer qu'à partir des années 1980, devenant par la suite un phénomène courant dans nos sociétés occidentales. Et bien que le $19^{\text {ème }}$ siècle ait vu arriver certaines affiches à caractère social, nous sommes 
à même de constater que celles-ci s'apparentaient bien plus à de la propagande qu'à des publicités défendant le bien commun. En effet, elles constituaient des revendications purement politiques. Nous pourrions nous demander si, de nos jours, de nombreuses campagnes sociétales n'auraient pas non plus une orientation politique, moins directe que celles du $19^{\text {ème }}$ siècle mais avec des idées sociales, économiques et culturelles palpables. Cette question mérite une étude spécifique que nous pourrions traiter dans un travail ultérieur, approfondissant ainsi nos recherches sur les publicités sociétales.

À la fin du $20^{\text {ème }}$ siècle, cette forme de sensibilisation a donc séduit tant les institutions publiques que les associations, entités qui exploiteront cette nouvelle tendance pour transmettre des messages, tout comme le font les compagnies privées, avec toutefois des intentions relativement distinctes. Ainsi, même si le schéma communicationnel, les stratégies discursives et les fonctions de ces deux types de publicité convergent pratiquement dans leur totalité -elles ont toutes deux pour rôles d'informer, persuader, promouvoir des valeurs sociales et finalement former des comportements- ce seront les objectifs visés et les retours espérés qui les distingueront l'une de l'autre. De ce fait, dans la publicité commerciale, les entreprises ont pour ambition de satisfaire des besoins individuels, ceux du client, tout en répondant aux intérêts mercantiles de l'entreprise qui se résument à faire des profits. Le fait de promouvoir des idéaux sociaux et de façonner des conduites a donc pour but de fidéliser la clientèle pour qu'elle achète le produit de manière itérative si possible. De son côté, la publicité sociétale répond au bien commun; il est question d'améliorer la qualité de vie en renforçant des comportements positifs ou en promulguant de nouvelles valeurs sociales de manière philanthropique. En résumé, s'oppose le bien collectif aux nécessités individuelles et l'enrichissement humain au profit monétaire.

Puis, un décalage conceptuel existe bel et bien entre ces deux types de discours. Selon C. Cossette dans son ouvrage La publicité, déchet culturel (2001), deux genres communicationnels s'opposent: un discours de sensibilisation propre aux publicités sociétales et un discours de consommation, typique des campagnes commerciales. En guise de récapitulatif des ressemblances et des disparités entre ces deux types d'objets publicitaires, nous reprendrons un tableau publié dans notre thèse datant de 2017: 
NATURE ET PUblicité SOCIALE: DU SCÉNARIO CATASTROPHE À LA RECONQUÊTE DE...

\begin{tabular}{|c|c|c|}
\hline $\begin{array}{l}\text { TYPES DE } \\
\text { PRODUITS }\end{array}$ & $\begin{array}{l}\text { PUBLICITÉ SOCIALE } \\
\text { Idees } \\
\text { Concepts } \\
\text { Valeurs }\end{array}$ & $\begin{array}{l}\text { PUBUCITÉ COMMERCIALE } \\
\text { Blens } \\
\text { Services }\end{array}$ \\
\hline $\begin{array}{l}\text { OBNECTIFS } \\
\text { GÉNERAUX }\end{array}$ & $\begin{array}{l}\text { Blen commun } \\
\text { Qualité de vie } \\
\text { À caractêre phillanthropi que et } \\
\text { ed uat } f\end{array}$ & $\begin{array}{c}\text { Blen individuel } \\
\text { Consommat on quant tat ve } \\
\text { A caractere mercant le } \\
\text { Promot on d'actes d' achat et de } \\
\text { val eurs consumer istes }\end{array}$ \\
\hline $\begin{array}{l}\text { OBIECTIFS } \\
\text { SPEEIFIQUES }\end{array}$ & $\begin{array}{l}\text { Informer } \\
\text { Persuader } \\
\text { Formater }\end{array}$ & $\begin{array}{l}\text { Informer } \\
\text { Falrevendre } \\
\text { Persuader } \\
\text { Fidel lser } \\
\text { Formater }\end{array}$ \\
\hline $\begin{array}{l}\text { SCHËMA DE } \\
\text { COMMUNICATION }\end{array}$ & \multicolumn{2}{|c|}{$\begin{array}{l}\text { Schéma unidirect onnel où un dlalogue est feint } \\
\qquad \text { Achat du produ it ou du service }\end{array}$} \\
\hline RÉTROACTIONS & \multicolumn{2}{|c|}{ Changement des comportements } \\
\hline $\begin{array}{l}\text { PROCËDÉS } \\
\text { SÉMIOTIQUES, } \\
\text { LINGUISTIQUES ET } \\
\text { ETHETRQUES }\end{array}$ & \multicolumn{2}{|c|}{$\begin{array}{c}\text { Discours basés sur Yémot on } \\
\text { Discours néo logil ques et plurilingues } \\
\text { Discours transgressifs, choquants, humorist ques } \\
\text { Communicat on par la marque } \\
\text { Miseen scène du réel } \\
\text { Ident f tat on au récepteur adolescent } \\
\text { Ef tet rhétorl que }\end{array}$} \\
\hline
\end{tabular}

Figure 1. Rapprochement entre publicité sociale et publicité. commerciale.

Ce schéma résume clairement les notions que nous venons de commenter et nous permet de mieux cerner les aspects linguistiques, sémiotiques, mais aussi discursifs, communicatifs, socio-économiques et culturels de la publicité sociale dans notre monde contemporain.

Avant d'aborder le thème de la nature en publicité, nous apporterons quelques précisions théoriques quant aux éléments sémio-linguistiques et discursifs présents dans les affiches sociales analysées. Nous observons ainsi que les deux campagnes objets de notre étude cherchent à provoquer des émotions chez le récepteur: la première au moyen de l'ironie et la seconde par le biais d'un discours rassurant. En effet, comme évoqué dans les écrits de I. Becheur et P. Valette Florence (2014), les concepteurs publicitaires tentent de toucher émotionnellement les destinataires afin de capter leur attention et de parfaire la communication commerciale et sociale. L'humour, vecteur émotionnel convaincant parmi tant d'autres, joue un rôle primordial dans la réussite d'une communication publicitaire:

Très prisé par la publicité actuelle, l'humour s'avère un moyen privilégié de tenter de persuader plus facilement les destinataires potentiels. Il consiste en un procédé énonciatif, producteur d'une anomalie, se manifestant en dehors des normes linguistico-communicatives, logiques ou sociales qui, pour être pris en considération, soit susciter plaisir et connivence chez le récepteur prédisposé au sérieux et au bon sens et contraint par la réalité (López Díaz, 2006: 119). 
Non seulement la campagne de 2011 recourt à l'ironie mais aussi à l'humour noir grâce à une violence des mots et des images parfois qui ne sont pas sans choquer et faire réfléchir les consommateurs. De plus en plus présent dans les campagnes sociales (Eguizábal, 2008), il permet effectivement une réponse affective et cognitive notable de la part du public. Dans la campagne de 2016, les affiches ironiques laissent place à des publicités plus sages mais non moins éloquentes. Jouant avec les sentiments empathiques des récepteurs, FNE nous propose des slogans optimistes accompagnés de photographies plaisantes. Au sujet de l'empathie, J.-C. Soulages met en avant qu'elle favorise généralement une identification du destinataire aux propos publicitaires: "en misant sur la complicité et l'empathie, le publicitaire s'attache à construire ce qui se donne comme une communauté discursive d'appartenance" (2006: 118). Dans les deux cas étudiés, d'autres procédés sémiotiques et linguistiques sont également employés afin de convaincre les individus de l'importance des messages environnementaux que souhaite transmettre l'association. Nous trouvons par exemple la présence de termes anglophones ou d'expressions argotiques, parlures de nos sociétés occidentales reprises fréquemment dans les discours publicitaires pour se rapprocher de la langue orale des individus, ainsi que de figures de rhétoriques qui se révèlent être de plus en plus courantes dans les campagnes publicitaires contemporaines et qui attirent indubitablement l'attention "because consumers know that they should seek out similarities when they encounter a visual template of this kind" "(Phillips et McQuarrie, 2004: 115). Comme le reflète le tableau exposé précédemment, il est clair que les mécanismes linguistiques, sémiotiques et discursifs utilisés dans les campagnes sociétales analysées dans ce travail sont identiques à ceux que nous pourrions déceler dans les affichages publicitaires commerciaux puisque plusieurs des objectifs que les entités souhaitent atteindre sont similaires à ceux des entreprises, à savoir informer, persuader et formater.

\subsection{La nature en publicité}

Les thématiques abordées dans les publicités sociales sont variées, toutefois les campagnes sociétales sont pour la plupart destinées à protéger les droits des individus et à sauvegarder l'environnement. Au cours de ce travail, nous nous intéresserons tout particulièrement à ce dernier domaine, qui n'est pas sans lien direct avec le premier cité, à savoir la protection de la personne. En effet, la population est confrontée à de nouvelles inquiétudes environnementales, problématiques qui se trouvent en étroite relation avec sa santé. L'intérêt général rejoint ici l'intérêt individuel qui est celui de jouir d'une bonne santé en adoptant un style de vie sain et respectueux de la nature.

De plus, comme le souligne très justement T. Maillet (2006, 2007), le consommateur manifeste plus que jamais son souhait de consommer de manière responsable et ce, pour

1 Parce que les consommateurs savent qu'ils doivent chercher des similarités quand ils font face à un modèle visuel de ce type (traduction personnelle). 
diverses raisons. Premièrement pour se libérer du sentiment de culpabilité qu'il éprouve au moment de l'achat, conscient de vivre dans une société de surconsommation. Deuxièmement, consommer écolo est une manière de se démarquer au niveau socio-économique. Il s'agit souvent de produits plus onéreux, c'est pourquoi leur acquisition permet de se différencier du reste de la population et d'avoir l'impression d'appartenir à un groupe social. Troisièmement, nous essayons de consommer bio par respect de nos convictions, nous souhaitons en réalité être consommateurs de produits non-nuisibles au développement durable:

Les préoccupations environnementales et sociales prennent une place de plus en plus importante dans la société. [] Le consommateur est de plus en plus soucieux d'une consommation responsable prenant ainsi en compte les conséquences de ses choix personnels sur la société dans son ensemble. [ ] Une consommation dite engagée se développe (Hérault, 2012: 8-9).

La multiplication des institutions et mouvements environnementaux est pour beaucoup dans cet engouement écologique. Nos sociétés occidentales font en effet face au développement d'initiatives pro-environnementales toute échelle confondue. La préservation de la nature préoccupe nos institutions publiques. C'est ainsi que de nouvelles politiques sont apparues prônant le respect de l'environnement: des protocoles internationaux, tels que celui de Kyoto ratifié officiellement en 2005 par la plupart des États membres de l'Organisation des Nations Unies puis l'Accord de Paris entré en vigueur en 2016, des directives européennes, des lois et décrets nationaux, des politiques locales, ces dernières étant plus aptes à agir sur le terrain (Garabuau-Moussaoui, 2011). Mentionnons ici l'importance des engagements internationaux qui, malgré des résultats mitigés ${ }^{2}$, sont amplement retransmis dans les médias, ce qui permet une sensibilisation accrue des populations. Outre l'augmentation de politiques publiques environnementales, les associations telles que FNE et mouvements privés défendant les mêmes causes prolifèrent depuis quelques années. Ainsi l'individu est-il mieux informé et plus sensible aux problèmes climatiques actuels par les nouvelles obligations légales et morales l'amenant à préférer un mode de vie respectueux de l'environnement.

Afin de répondre aux attentes des consommateurs, l'argument écologique dans la publicité contemporaine est, par conséquent, de plus en plus présent, tant dans les campagnes sociétales que les commerciales. En ce qui concerne ces dernières, plusieurs moyens sont employés par les publicistes pour rendre leurs objets publicitaires plus "nature". On peut en souligner quelques-uns tels que donner un nom à connotation environnementale au produit ou à la marque comme pour l'entreprise spécialisée dans les soins cosmétiques Biotherm ou recourir à des slogans accrocheurs comme "Naturellement pulpeuse" pour Orangina ou

2 Signé le 11 décembre 1997, le Protocole de Kyoto a pour objectif d'assurer la réduction des émissions de gaz à effet de serre dans les pays signataires. En dépit de résultats prometteurs dans les pays de l'Union Européenne notamment, d'autres comme la Chine n'ont, pour beaucoup, pas fait les efforts nécessaires. Ce traité ne fut par ailleurs jamais ratifié par les États-Unis, pays qui, en 2017, décida de surcroît de sortir de l'Accord de Paris. 
"Pure, à la roche minérale, $48 \mathrm{~h}$, sans alcool" pour Rexona. Sur le plan sémiotique, l'image peut évoquer la nature, représentation le plus souvent truquée pour vendre cette idée de produit naturel. L'emballage avec une symbolique écologique est également un des moyens les plus exploités. Remarquons en guise d'exemple la marque Danone avec son yaourt nommé "bio" au conditionnement de couleur verte, tonalité représentative de la nature en général. Il s'agit ici d'assimiler la marque au respect de l'environnement bien que l'usage d'emballages plastiques ne puisse que nuire au développement durable. Enfin, l'apparition de logo écologique sur l'empaquetage ou dans la propre publicité commerciale est de la même manière un procédé récurrent. En effet, l'association d'un produit à des organismes luttant contre les problèmes environnementaux est devenue un phénomène en vogue.

Toutes ces méthodes de marketing sont vigoureusement pointées du doigt par les organisations environnementales. L'expression greenwashing a justement été créée à cet effet: elle dénonce les agences de publicité qui utilisent de manière abusive et mensongère l'argument écologique afin de répondre aux attentes des consommateurs de plus en plus éco-responsables. Ce mécanisme, également nommé écoblanchiment ou verdissage, est d'ailleurs depuis 2008 sous la surveillance de l'Autorité de régulation professionnelle de la publicité (ARPP). Cet organisme, en étroite collaboration avec le ministère de l'Écologie, de l'Énergie et du Développement Durable, a pour mission entre autres de publier annuellement un bilan Publicité \& Environnement. Ces études rendent compte du niveau d'application par les entreprises des règles d'éthique recommandées par l'ARPP quant au développement durable. Des données concrètes nous sont ainsi fournies: des 736 visuels liés à l'environnement visionnés en 2017, 94\% sont conformes aux normes, 5\% constituent un manquement car ils incitent à un comportement non éco-responsable ou induisent en erreur le récepteur et $1 \%$ font l'objet de réserves (Martin, 2017). Il s'agit de statistiques encourageantes qui ne sont pas sans masquer un phénomène majeur, à savoir les écarts bien plus conséquents commis dans les publicités émises sur les réseaux sociaux tels que l'emploi de visuels exagérés, le manque de nuances dans les messages et l'abus de mot-dièses ou d'hyperliens fallacieux. Par ailleurs, l'analyse effectuée par l'ARPP et retranscrite dans ses bilans a ses limites puisque seul un échantillon réduit des objets publicitaires diffusés sur les réseaux sociaux s'avère être examiné. Les messages abusifs de ces nouveaux médias sont donc certainement bien plus nombreux que ce que nous laisse penser le résumé publié par cet organisme. Or, comme nous le confirment les résultats de nos sondages, les individus consultent le plus souvent les campagnes publicitaires sur les pages internet et les réseaux sociaux ( $c f$. paragraphe 3.2 de ce travail).

En somme, la nature est un thème surexploité par les annonceurs tentant de capter l'attention du consommateur à partir de sujets qui le touchent d'une certaine manière. Les campagnes commerciales, mais aussi sociétales, foisonnent donc d'objets publicitaires mettant en avant la protection de l'environnement. Ajoutons à ceci le fait que 53,2\% des personnes 
NATURE ET PUblicité SOCIALE: DU SCÉNARIO CATASTROPHE À LA RECONQUÊTE DE...

sondées affirment que les publicités sociales influencent "normalement" leur comportement ( $c f$. Figure 3). Pour toutes ces raisons, il nous a paru pertinent d'analyser les deux campagnes de FNE et de contraster les procédés linguistiques et sémiotiques employés dans celles-ci afin de déterminer quel type de publicité sociale est plus à même de déclencher des sentiments forts chez ses récepteurs.

\section{3. Étude contrastive des deux campagnes de France Nature Environnement}

La seconde partie de notre travail se divise en deux sections qui se complémentent l'une et l'autre. Dans un premier temps, nous observerons les éléments linguistiques et sémiotiques des affiches de FNE afin de mettre en exergue les différences probantes entre ses deux campagnes datant respectivement de 2011 et 2016. Dans un second temps, nous analyserons et interpréterons les résultats obtenus des enquêtes menées récemment concernant l'impact de ces dites campagnes sur les récepteurs francophones.

\subsection{Analyse sémio-linguistique des affiches}

Notre corpus ${ }^{3}$ comprend un ensemble de quinze visuels publiés en 2011 et 2016. Nous analyserons les éléments linguistiques et sémiotiques qui le définissent afin de présenter des considérations discursives pertinentes. La terminologie, la phraséologie et les caractéristiques discursives des deux campagnes seront contrastées dans le but de déterminer les aspects microtextuels de chacune d'entre elles. De la même manière, ces affiches seront examinées depuis une perspective pragmatique afin d'identifier les stratégies persuasives et leurs résultats opérationnels.

La première campagne de 2011 regroupe un ensemble de six affiches et a pour unique thématique les excès de l'agriculture industrielle. Elles suivent toutes le même schéma conceptuel: une illustration suggestive occupant la totalité de l'affiche, une accroche brève et provocante en lettres majuscules suivie d'une ou deux phrases complètes ${ }^{4}$, les deux en haut de l'affiche; puis une invitation à se sensibiliser grâce à la formule "Agissez avec nous" suivie de l'adresse de la page de l'organisme et du logo pour que le récepteur puisse s'y connecter et y collecter de plus amples informations. Les slogans sont les suivants:

\section{ARRETTEZ VOS SALADES}

L'élevage industriel des parcs et les engrais génèrent des algues vertes. Leur décomposition dégage un gaz mortel pour l'homme.

3 Les affiches des deux campagnes sont disponibles sur le site de l'association FNE $<$ https://www.fne.asso.fr/ $>$ [15/05/2020].

4 Nous entendons par phrase complète ici un énoncé comprenant sujet, verbe et complément, correct au niveau grammatical. 
BONNES VACANCES

L'élevage industriel des parcs et les engrais génèrent des algues vertes. Leur décomposition dégage un gaz mortel pour l'homme.

C'EST SANS DANGER

Concernant les OGM, on n'a pas encore assez de recul...

FIN DU BUZZZZZ

Certains pesticides présentent un danger mortel pour les abeilles et ce n'est pas du cinéma.

GROS MENTEUR

La loi n'impose pas l'étiquetage des viandes issues d'animaux nourris aux OGM.

KILL BEES (traduit en bas de l'affiche par tueurs d'abeilles)

Certains pesticides présentent un danger mortel pour les abeilles et ce n'est pas du cinéma.

Nous remarquons dans l'ensemble de ces slogans l'emploi de l'ironie permettant la transmission d'un message puissant et provocateur. En effet, comme nous l'indique judicieusement J.-L. León (2001), les trois types humoristiques publicitaires - ironie, satire et parodie - sont couramment utilisés en publicité afin d'attirer l'attention des récepteurs, friands de campagnes percutantes.

Soulignons tout d'abord que tant l'école psychanalytique freudienne que l'école morale selon $\mathrm{H}$. Bergson ou les théories de la fin de $21^{\text {ème }}$ siècle centrées sur le récepteur coïncident sur le fait que l'humour a pour objectifs principaux la suppression des obstacles de communication entre l'entreprise ou l'organisme et les destinataires des publicités ainsi que la création de liens émotionnels entre eux (Dorey et Zollinger, 2000). R. Eguizábal rajoute à ceci que "el humor publicitario - como todo el humor actual - evacua la negatividad" (2007: 298). En d'autres termes, le facteur comique facilite l'atténuation du pessimisme ambiant et, dans le cas des publicités sociales, il permet de relativiser la violence et l'alarmisme de certains messages. Par ailleurs, ce même auteur met également en avant le fait que l'humour joue un rôle essentiel dans l'accès au style de vie détaché et hédoniste qui caractérise le monde dans lequel nous développons notre identité personnelle et sociale aujourd'hui:

Ha progresado un estilo desenfadado e inofensivo, sin negación ni oposición a nada, sin contenidos, sin profundidad, característico de los medios de comunicación, que se ha instalado en la vida cotidiana [...] El ascenso del humor es parte del proceso de relajamiento de las formas, las formalidades están ahora muy mal vistas, todo debe ser cordial, campechano, 'espontáneo'; los ritos y las ceremonias pierden su sitio, es la cultura hedonista ${ }^{6}$ (Ibid.: 297-300).

5 L'humour publicitaire - comme tout l'humour actuel - évacue la négativité (traduction personnelle).

6 Un style désinvolte et inoffensif, sans négation ni opposition, sans contenus, sans profondeur, caractéristique des moyens de communication, a prospéré, un style qui s'est installé dans la vie quotidienne [...] L'ascension 
La comicité du message permet donc d'attirer l'attention des récepteurs des publicités, nécessiteux de discours épicuriens. Enfin, Y. Zhang (1996) insiste sur le fait que l'humour réussi est un facteur influençant favorablement à la fois l'attitude face à la publicité et l'attitude face à la marque et, par extension, il pourrait influencer positivement le comportement à l'égard de celle-ci. En ce qui concerne les publicités sociétales et plus concrètement la campagne de FNE diffusée en 2011, l'effet comique permet donc d'attirer l'attention des populations et de mieux les sensibiliser aux problèmes liés à l'environnement.

Revenons-en désormais à l'ironie, style humoristique le plus répandu en publicité, objet d'étude des six slogans cités précédemment et ainsi décrite par J.-L. León:

La ironía sirve para conseguir la atención del receptor del mensaje, al introducir un contraste con valor de comicidad. Es patente la superioridad atencional y persuasiva de la ironía sobre mensajes más directos y convencionales y resulta apropiada incluso en temáticas caracterizadas por su gravedad, como es el consumo de alcohol y drogas ${ }^{7}$ (2001: 138).

Il en est de même pour ces six affiches défendant une agriculture respectueuse de l'environnement et de l'homme: un ton ironique est utilisé afin de masquer le sérieux des motifs de cette campagne.

Dans la première affiche au slogan familier “ARRÊTEZ VOS SALADES. L'élevage industriel des parcs et les engrais génèrent des algues vertes. Leur décomposition dégage un gaz mortel pour l'homme", l'association algues/salades est intéressante et de cette dernière émane l'ironie, tant au niveau textuel que visuel, étant donné la ressemblance frappante des algues et des laitues que nous consommons. En effet, la photo nous enseigne un élément vert, couleur censée donner une image de produit sain alors qu'en réalité, ces algues sont mauvaises pour la santé et l'environnement. Mentionnons ici l'emploi d'une première phrase d'accroche argotique "ARRÊTEZ VOS SALADES" utilisée généralement pour interrompre une personne tentant de nous tromper avec son discours et qui aide à interpeller le récepteur de l'affiche.

La deuxième affiche dénonçant également les dangers résultant de l'élevage industriel des porcs et de l'utilisation abondante d'engrais a pour accroche initiale "BONNES VACANCES". Elle est accompagnée d'une image choc d'un petit garçon jouant dans et avec les algues vertes, comme le ferait tout autre enfant avec le sable lors de vacances sur le littoral.

de l'humour fait partie du processus de relâchement des formes, les formalités sont désormais très mal vues, tout doit être avenant, bon enfant et 'spontané'; les rites et les cérémonies perdent leur place, c'est la culture hédoniste (traduction personnelle).

7 L'ironie sert à capter l'attention du récepteur du message, en introduisant un contraste à valeur comique. La supériorité attentionnelle et persuasive de l'ironie sur les messages plus directs et conventionnels est manifeste et elle résulte être seyante même pour les thèmes graves, comme l'est la consommation d'alcool et de drogue (traduction personnelle). 
L'ironie issue de l'association de cette formule avec l'image déplaisante permet indéniablement de commotionner et sensibiliser la population.

Dans la troisième affiche, accompagnant le slogan "C'EST SANS DANGER Concernant les OGM, on n'a pas encore assez de recul...", nous faisons face à un visuel choc de nouveau, à savoir la photo d'un jeune homme portant un pistolet-épi de maïs à sa tempe. Une double contradiction permettant l'ironie est à souligner. Premièrement l'image de l'individu simulant son suicide avec un épi de maïs épousant la forme d'une arme à feu est à l'opposé de l'accroche “C'EST SANS DANGER". Deuxièmement, cette même image est contraire à l'expression "avoir du recul” puisque l'arme-épi de maïs est disposée contre la tempe du jeune homme, donc sans aucun recul. L'image violente est ici atténuée grâce à l'ironie entre les composants linguistiques et les éléments sémiotiques, toutefois un clair message est transmis: l'association FNE réussit au moyen de cette figure de style à dénoncer les conséquences néfastes des OGM pourtant présents dans de nombreux aliments que nous consommons.

Pour l'affiche au slogan "FIN DU BUZZZZZ. Certains pesticides présentent un danger mortel pour les abeilles et ce n'est pas du cinéma", les concepteurs ont eu recours à deux procédés captant nécessairement l'attention des individus. Le premier est la répétition d'un même caractère dans l'onomatopée faisant référence au son émis par les abeilles, avec la présence de la lettre $z$ cinq fois à la suite. Le second est justement l'emploi de ce terme anglophone buzz qui renvoie à la notion de phénomène commercial ou social manifeste. En effet, signifiant bourdonnement ou rumeur en langue anglaise, cet anglicisme recouvre selon le dictionnaire en ligne Larousse deux définitions: (1) "Forme de publicité dans laquelle le consommateur contribue à lancer un produit ou un service via des courriels, des blogs, des forums ou d'autres médias en ligne; bouche-à-oreille" et (2) "Rumeur, retentissement médiatique, notamment autour de ce qui est perçu comme étant à la pointe de la mode (événement, spectacle, personnalité, etc.)"8. Employer ce terme dans l'expression "FIN DU BUZZZZZ" n'est pas sans évoquer un parallélisme ironique entre la mort des abeilles et la fin d'un phénomène de mode.

Dans la cinquième affiche à la formule tapageuse "GROS MENTEUR. La loi n'impose pas l'étiquetage des viandes issues d'animaux nourris aux OGM", l'association FNE pointe du doigt l'étiquetage mensonger qui séduit indubitablement le consommateur, avide de produits naturels car désireux de consommer de manière responsable tant pour le respect de l'environnement que pour sa santé ( $c f$. paragraphe 2.2 de ce travail).

Enfin, la sixième affiche rapportant également le danger des pesticides sur les abeilles emploie cette fois un slogan se référant à un film d'action américain réalisé par Q. Tarantino et sorti en deux parties en 2003 et 2004. Tant la formule linguistique "KILL BEES" renvoyant au titre Kill Bill, que l'ensemble de l'affiche employant les couleurs phares du film - jaune, noir et une touche de rouge - assurent la référence que l'organisme souhaite transmettre aux

$8<$ https://www.larousse.fr/dictionnaires/francais/buzz/10910386> [30/05/2020]. 
NATURE ET PUblicité SOCIALE: DU SCÉNARIO CATASTROPHE À LA RECONQUÊTE DE...

destinataires des messages. Cette œuvre cinématographique, rendant hommage aux films de samouraïs et de spaghettis westerns, est d'ailleurs réputée pour ses scènes de violence, ce qui n'est pas sans produire un effet ironique lorsque celle-ci est utilisée pour se reporter à l'extermination des abeilles pour cause d'abus de pesticides dans notre système agricole.

La deuxième campagne intitulée "Vivre dans un monde vivable" et datant de 2016 a adopté une toute autre stratégie de communication plus légère et bien moins offensive. Pourvue de neuf affiches, elle rend cette fois compte de divers problèmes liés à l'environnement et ne se centre plus seulement sur les dangers de l'agriculture industrielle. Les visuels possèdent tous la même structure: neuf photos idylliques représentant des slogans porteurs d'espoir situés au centre de l'affiche:

Avoir du beau temps et pas le pic de pollution qui va avec.

Courir en ville sans penser à ce que l'on respire.

Pouvoir faire ses courses sans lire la composition du moindre truc que l'on achète.

Pouvoir mordre dans une pomme sans réfléchir.

Pouvoir se baigner dans une rivière.

Pouvoir se dire qu'un paysage était là avant nous et qu'il sera encore là après.

Revoir des abeilles.

Utiliser une crème hydratante pendant des années sans découvrir plus tard que l'on aurait pas dû.

Voir réapparaître ce qui est en voie de disparition.

Dans la première affiche au slogan évocateur "Avoir du beau temps et pas le pic de pollution qui va avec", FNE met en avant un problème persistant dans toutes les grandes agglomérations, à savoir la pollution. S'adressant au public français, une photo de la ville de Paris sert de visuel, mais l'acteur principal de cette affiche reste le ciel. Précisons que la ville de Paris ainsi que la Préfecture de Police ont pris des mesures depuis les années 1990 pour réduire la pollution dans l'une des villes les plus denses au monde telles qu'évaluer la qualité de l'air, rendre le stationnement résidentiel gratuit pour privilégier les transports en commun, rendre l'abonnement quotidien pour le Vélib' partiellement gratuit et réduire la vitesse maximale autorisée, entre autres ${ }^{9}$. Cependant ces actions ne suffisent malheureusement pas, d'où l'importance d'une campagne de ce type pour sensibiliser au mieux la population. Par ailleurs, l'opposition présente dans cette courte formule entre avoir du beau temps et pic de

$9<$ https://www.paris.fr/pages/mesures-prises-en-cas-de-pic-de-pollution-2720> [31/05/2020]. 
pollution grâce à la négation et pas est ici très intéressante. Ce contraste n'en est finalement pas un puisque les concepteurs choisissent d'allier ces deux idées en émettant une corrélation significative au moyen de l'expression qui va avec.

Ensuite, nous constatons que les accroches "Courir en ville sans penser à ce que l'on respire", "Pouvoir faire ses courses sans lire la composition du moindre truc que l'on achète", "Pouvoir mordre dans une pomme sans réfléchir" et "Utiliser une crème hydratante pendant des années sans découvrir plus tard que l'on aurait pas dû" suivent le même modèle syntaxique grâce à la préposition sans placée juste avant les verbes penser, lire, réfléchir et découvrir à l'infinitif. Il est question de quatre verbes impliquant des actions intellectuelles de l'interlocuteur, faire usage de son esprit et prendre connaissance de quelque chose. Avec ces verbes se trouvant juste après la préposition marquant l'absence de circonstances, l'association FNE transmet ici l'idée selon laquelle il est difficilement possible de vivre en accord avec la nature sans songer auparavant aux répercussions que nos faits et gestes peuvent impliquer.

Puis, dans les formules "Pouvoir se baigner dans une rivière", "Revoir des abeilles" et "Pouvoir se dire qu'un paysage était là avant nous et qu'il sera encore là après", les verbes à l'infinitif renvoient à la notion de faire de nouveau quelque chose qui nous est pour le moment impossible. L'énoncé "Pouvoir se baigner dans une rivière" sous-entend en effet qu'aujourd'hui nous ne pouvons profiter d'une baignade sans risque dans nos cours d'eau et la phrase "Revoir des abeilles" suggère que cet insecte a pour l'heure disparu de nos espaces naturels.

Enfin, le dernier slogan "Voir réapparaître ce qui est en voie de disparition" est, selon nous, de toutes les formules de la campagne, la plus séduisante de par la présence d'une antithèse à partir d'une figure dérivative. Les concepteurs de l'accroche ont en effet eu recours à une opposition rendue possible grâce à deux termes appartenant à la même famille, réapparaître et disparition, une stratégie discursive qui permet d'insister sur le propos en question. Ceci n'est pas sans rappeler la signature de la campagne "Vivre dans un monde vivable" exploitant également une figure de dérivation, à savoir la polyptote, en employant des variantes flexionnelles du même terme.

Somme toute, FNE nous présente deux campagnes foncièrement différentes, tant au niveau linguistique que sémiotique, qui ne sont pas sans attirer l'attention des consommateurs éco-responsables que nous sommes devenus, inquiets pour notre santé et pour le futur de notre environnement. En premier lieu, la campagne de 2011 nous rappelle qu'une étroite relation persiste entre la violence et l'humour en publicité: “el comportamiento transgresor publicitario debe ir necesariamente teñido de humor para ser aceptable, sólo así puede llegar a retratar los enfrentamientos ${ }^{10}$ " (León, 2001: 118). La transgression et l'effet comique sont effectivement deux recours stylistiques complémentaires. Il est de ce fait inhabituel de constater la présence de thématiques transgressives sans que celles-ci ne soient accompagnées d'ironie afin de rendre plus plaisant le message publicitaire et, à terme, séduire ses

10 Le comportement transgressif publicitaire doit nécessairement être teint d'humour pour devenir recevable, seulement ainsi il peut réussir à dépeindre les affrontements (traduction personnelle). 
destinataires. En outre, ce genre humoristique, tout comme la parodie et la satire, répond au besoin de décontraction des individus or le recours au comique rend possible cette légèreté discursive. Considéré moyen socio-affectif influent, l'humour est par conséquent fortement présent dans les campagnes publicitaires contemporaines et procure le plus souvent une sensation de bien-être chez les individus. En second lieu, les visuels à connotations positives de la campagne de 2016 assurent la persuasion des populations actuelles, dans le besoin de discours rassurants pour affronter leur quotidien parfois stressant et faire face aux différents problèmes sociaux, économiques ou environnementaux, entre autres. Ces neuf affiches laissent ainsi présager un avenir prometteur au moyen de stratégies discursives emphatiques, comme celles mentionnées auparavant. Autant la campagne datant de 2011 cherche à réveiller l'opinion publique et institutionnelle au moyen d'affiches "coup de poing", autant celle de 2016 tente de sensibiliser une audience plus importante en évitant les scénarios catastrophes.

Ce changement de stratégie marketing n'est pas anodin:

Pour toucher le public le plus large possible et sensibiliser même les moins acquis à la cause écologique, la campagne rappelle que si le combat de FNE est centré sur la préservation de la nature et de l'environnement, c'est au fond pour l'Homme, comme en faisant partie intégrante, que l'association se bat. Pour que chacun se sente pleinement concerné sans jouer sur les peurs, la campagne prend le parti d'éviter certains ressorts écologiques 'traditionnels': elle ne brandit aucun risque de catastrophe future ou lointaine et s'inscrit dans le cadre de vie quotidien et actuel des Français [...] Après la refonte de son site internet, de sa communication digitale et le lancement de son nouveau logo, cette campagne média est une étape de plus dans la montée en puissance de sa communication: en effet, le mouvement rassemblant plusieurs centaines de milliers de militants, bénévoles ou salariés, à travers les 3500 associations dont elle est le porte-parole, a décidé de passer à un niveau de visibilité et de notoriété supérieur ${ }^{11}$.

Traitant de préoccupations proches des réalités des individus, cette réorientation stratégique est jugée comme plus efficiente par ses promoteurs car plus "humaine". Alors que la première série d'affiches datant de 2011 dérange et a même fait l'objet de plaintes auprès de la Justice, la campagne "Vivre dans un monde vivable" est axée sur une communication centrée sur l'humain et ses expériences de vie. Le sondage réalisé en 2020 et son analyse postérieure figurant dans le paragraphe à suivre nous permettra de vérifier ces affirmations.

\subsection{Impact sur les récepteurs}

Nous avons lancé un questionnaire ${ }^{12}$ par le biais de la plateforme Google Formulaires le 17 mai 2020 afin d'apprécier laquelle de ces deux campagnes est la plus à même d'avoir une incidence sur la population francophone. Les 94 réponses que nous avons reçues nous

$11<$ https://www.fne.asso.fr/communiques/france-nature-environnement-part-en-campagne-pour-un-monde-vivable $>[04 / 06 / 2020]$.

12 Une transcription de ce questionnaire se trouve en Annexe $\mathrm{N}^{\mathrm{o}} 1$. 
permettent d'émettre de premiers résultats quant aux préférences sémio-linguistiques des individus concernant les publicités à caractère sociétal.

Constitué de quinze questions pour la plupart à choix unique, ce sondage commence avec deux variables socio-démographiques sur l'identité de genre et l'âge qui nous semblaient essentielles pour une étude de ce type. Il est manifeste que pour des raisons d'ordre social, les individus qui ont le plus répondu à cette enquête sont âgés de 20 à 60 ans. Toutefois, après avoir examiné en profondeur les réponses fournies selon l'âge et le genre, nous en sommes arrivés à la conclusion suivante: ces deux variables ne changent que très peu les conclusions générales que nous tirerons de ce questionnaire, c'est pourquoi nous avons décidé de ne pas en tenir compte pour le moment. Cependant, il nous semble judicieux de considérer ces données sociales pour de prochaines investigations qui étofferont notre présent travail.

Par la suite, nous avons émis cinq questions sur les publicités commerciale et sociale: leur influence sur les comportements des individus, le lieu de prédilection pour s'attarder sur ces publicités et le degré d'impact d'anciennes publicités sociétales sur les personnes sondées. Nous remarquons en premier lieu que l'emprise des publicités sur les récepteurs est la même qu'il s'agisse de campagnes commerciales ou sociales.
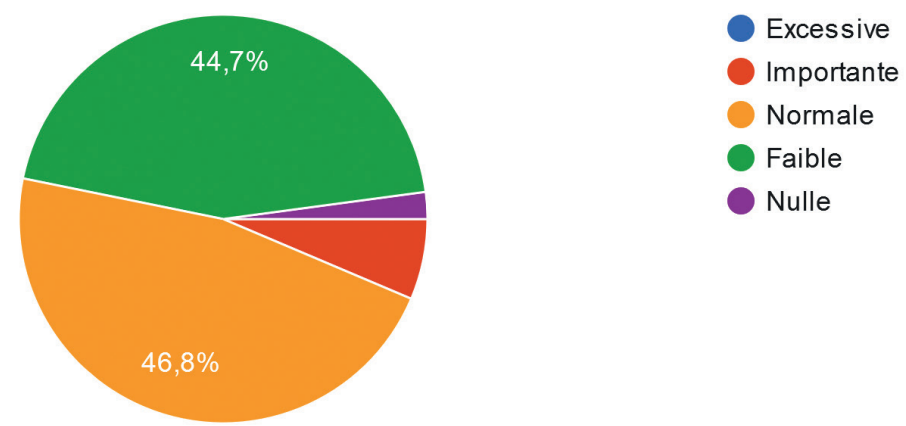

Figure 2. Quelle est l'influence des publicités commerciales sur vos achats?
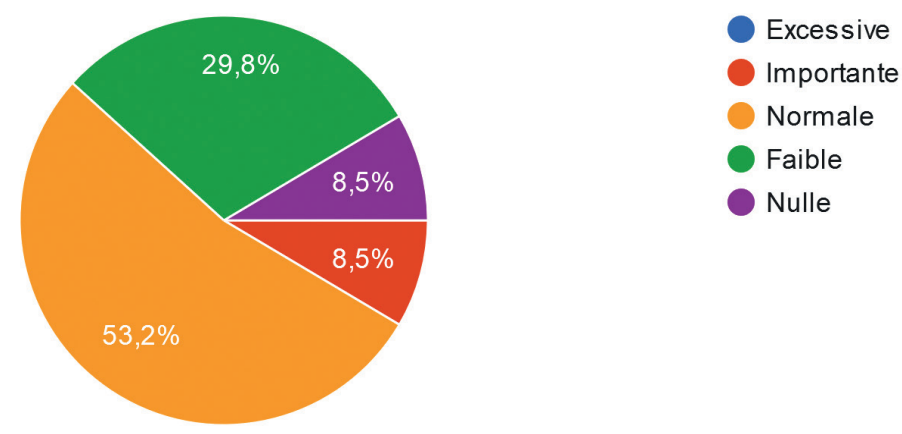

Figure 3. Quelle est l'influence des publicités sociales sur vos comportements? 
Dans les deux cas, aucune réponse située dans les extrêmes n'a été sélectionnée et la plupart ont répondu que l'emprise de ces deux types publicitaires était en majeure partie normale ou faible. Dans un sondage réalisé en 2013 dans les collèges et lycées français auprès de 1040 élèves, nous avions déjà détecté que la plupart des adolescents considéraient que les objets publicitaires les influençaient de manière normale dans leurs achats (Abraham, 2017), des données qui coïncident avec les précédentes malgré une tranche d'âge différente.

À la question "Généralement, où êtes-vous la/le plus à même de vous attarder sur des publicités?", 51,1\% ont répondu sur les pages internet et les réseaux sociaux, 29,8\% dans les médias traditionnels et $12,8 \%$ dans l'espace urbain, le publipostage et la presse ou les magazines écrits n'ayant été que très peu sélectionnés. Les nouveaux médias sont donc les plus aptes à transmettre des messages tant commerciaux que sociaux et les plus enclins à diffuser des informations mensongères ( $c f$. paragraphe 2.2 de ce travail). Dans le cas de FNE, les deux campagnes ont surtout profité d'une diffusion dans le milieu urbain, bien que les affiches soient également disponibles sur internet, que ce soit sur le propre site de l'association ou sur d'autres pages web ${ }^{13}$. Cela nous amène à la réflexion suivante: une publication de ces messages sur les réseaux sociaux ne serait-elle pas plus efficace pour sensibiliser un plus grand nombre d'individus?

Puis à la question "Certaines campagnes de publicités sociales vous ont-elles tout particulièrement marqué(e)?”, 42,6\% admettent que oui, quelques-unes bien qu'une part importante des personnes sondées admettent que non $(31,9 \%)$, des statistiques quelque peu alarmantes qui montrent en fin de compte que les publicités sociétales n'ont pas la portée espérée. En outre, des individus ayant répondu à l'affirmative, la plupart cite comme campagnes frappantes celles de la prévention et sécurité routières (46\%), les autres thèmes étant en dessous de la barre des $20 \%$ (publicités contre le tabagisme, contre la violence faite aux femmes, pour les ONG médicales et d'aide humanitaire, pour les associations environnementales, etc.).

Enfin, nous avons centré nos huit dernières questions sur les campagnes de l'association FNE. Premièrement, nous avons souhaité savoir quel était le pourcentage de personnes sondées connaissant le dit organisme ainsi que les deux campagnes objets de notre étude. À notre grande surprise, seul $12,8 \%$ avaient déjà entendu parler de l'association et $6,4 \%$ avaient déjà pris connaissance des affiches. Ces données nous enseignent un paramètre non négligeable à prendre en compte pour l'analyse des prochains résultats: la plupart des personnes interrogées auront vu ces posters publicitaires pour la toute première fois lorsqu'il auront complété le questionnaire.

Nous les avons ensuite interrogés sur leurs préférences en matière de publicité, tout particulièrement entre ces deux campagnes.

$13<$ https://www.fne.asso.fr/communiques/la-campagne-de-france-nature-environnement-censur\%C3\%A9e > et $<$ https://www.fne.asso.fr/communiques/france-nature-environnement-part-en-campagne-pour-un-monde-vivable $>[15 / 05 / 2020]$. 


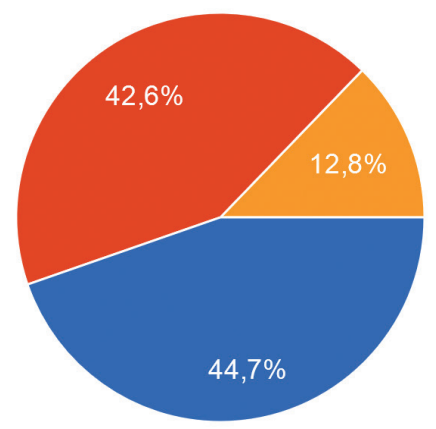

Campagne contre l'excès de l'agriculture industrielle (2011)

Campagne "Vivre dans un monde vivable" (2016)

Je n'ai pas de préférence.

Figure 4. L'association FNE a lancé deux campagnes: la première contre l'excès de l'agriculture industrielle en 2011 et la seconde en 2016 intitulée "Vivre dans un monde vivable". Laquelle préférez-vous?

La première question, plus générale, nous a permis de constater que les réponses étaient mitigées: $44,7 \%$ ont sélectionné la campagne contre l'excès de l'agriculture industrielle, $42,6 \%$ celles de "Vivre dans un monde vivable" et 12,8\% ont signifié ne pas avoir de préférences entre les deux. Puis, nous avons désiré identifier quels étaient les aspects des deux campagnes qu'ils avaient le plus et le moins apprécié.
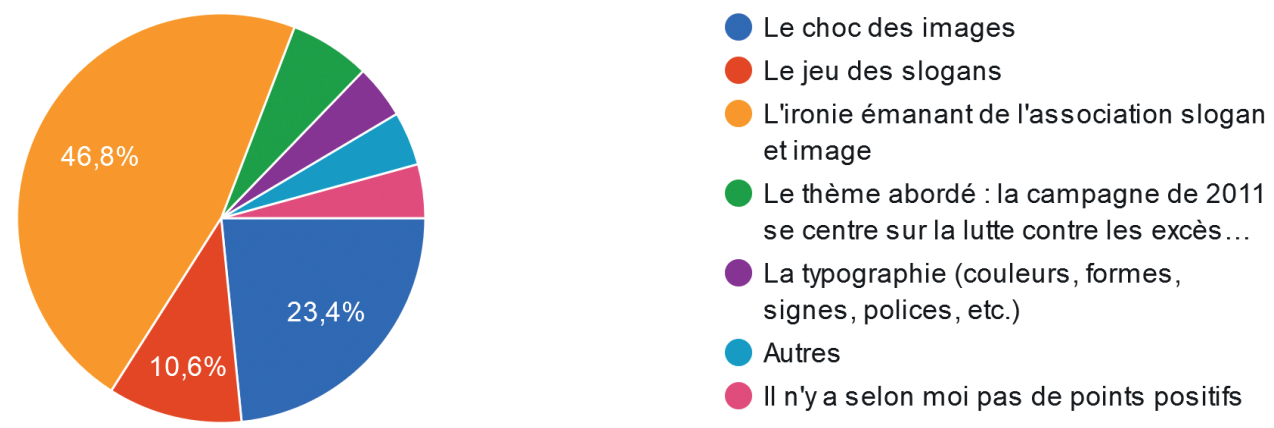

Figure 5. Quel est, selon vous, le point positif de la campagne contre l'excès de l'agriculture industrielle (2011)? 


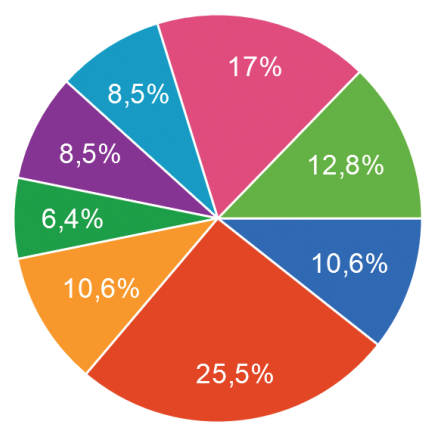
La violence des images
La longueur des slogans
L'ironie émanant de l'association slogan et image
Qu'il s'agisse d'une campagne à sujet unique
La typographie (couleurs, formes, sign...
L'absence de "nom de campagne"
Autres
II n'y a selon moi pas de points négatifs

Figure 6. Quel est, selon vous, le point négatif de la campagne contre l'excès de l'agriculture industrielle (2011)?

D'une part, il est clair que pour la campagne de 2011, l'élément qui a avant tout séduit les récepteurs est l'ironie émanant de l'association slogan et image (46,8\%), suivi du choc des images $(23,4 \%)$, et du jeu des slogans $(10,6 \%)$. Mais les slogans sont aussi le composant sémio-linguistique qui a récolté le plus d'évaluations négatives étant donné que $25,5 \%$ des personnes sondées les estiment trop longs. Néanmoins, retenons que la campagne basée sur l'ironie est une réussite puisque c'est justement cet aspect qui a le plus marqué l'esprit des personnes interrogées. Comme nous l'avons énoncé auparavant, l'ironie camoufle le sérieux du thème abordé tout en choquant les individus; dans ce cas de figure, au moyen d'accroches et d'images saisissantes, pouvant aller jusqu'à offenser les plus sensibles. Il est finalement question d'une technique publicitaire permettant une meilleure mémorisation des campagnes et donc une plus grande conscientisation des populations ( $c f$. paragraphe 3.1 de ce travail).
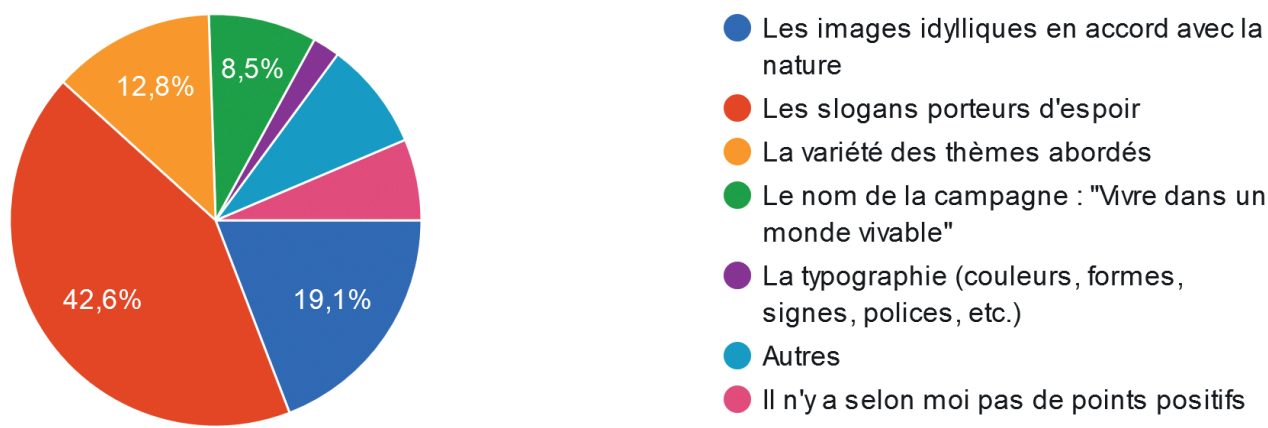

II n'y a selon moi pas de points positifs

Figure 7. Quel est, selon vous, le points positif de la campagne "Vivre dans un monde vivable" (2016)? 


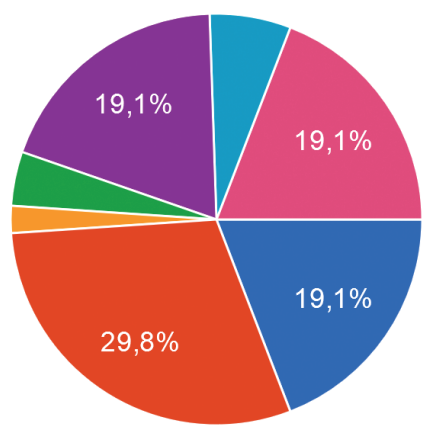

La longueur des slogans

Les images lisses et bienveillantes

La variété des thèmes abordés

Le nom de la campagne : "Vivre dans un monde vivable"

La typographie (couleurs, formes, signes, polices, etc.)

Autres

II n'y a selon moi pas de points négatifs

Figure 8. Quel est, selon vous, le point négatif de la campagne "Vivre dans un monde vivable" (2016)?

D'autre part, de la campagne "Vivre dans un monde vivable", ce sont les slogans porteurs d'espoir que les individus interrogés ont le plus appréciés $(42,6 \%)$, suivi des images idylliques en accord avec la nature $(19,1 \%)$ et de la variété des thèmes abordés $(12,8 \%)$. Rappelons que la campagne précédente se centrait sur une unique thématique, ce qui n'avait pas nécessairement attiré l'attention des récepteurs, que ce soit positivement ou négativement ( $c f$. Figure 4 et 5). Notons également que l'item "nom de campagne" a été sélectionné par 8,5\% des personnes sondées, or la campagne de 2011 était dépourvue de signature rassemblant les affiches. Parmi les points négatifs mis en avant pour cette deuxième série publicitaire, $29,8 \%$ considèrent que les images lisses et bienveillantes ne favorisent pas la campagne, puis 19,1\% remettent en cause la typographie et une même proportion d'individus questionnent la longueur des slogans. Par ailleurs, soulignons ici que 19,1\% estiment qu'il n'y a pas de points négatifs, contre 12,8\% pour la campagne précédente. En définitive, les affiches de 2016 sont clairement appréciées pour la teneur des slogans même si des formules plus brèves seraient valorisées. Les images tendres et harmonieuses n'ont, au contraire, pas obligatoirement convaincu les destinataires de cette campagne.

En résumé, les deux techniques marketing ont réussi à atteindre deux publics distincts, le premier attiré par le style publicitaire $\operatorname{choc}^{14}$ et le second par des affiches promettant un meilleur avenir. Il est question de deux stratégies fondamentalement opposées qui permettent toutefois de capter l'attention de la population actuelle, partageant une culture certes, mais présentant des goûts hétéroclites. Lors de l'analyse de la première campagne, nous avons déjà évoqué l'influence de l'humour et tout particulièrement de l'ironie dans les objets publicitaires contemporains. M. Bonhomme et S. Pahud confirment d'ailleurs cet impact sur l'individu dans leur article "Un renouveau actuel de la rhétorique publicitaire?":

14 La publicité choc, ou shockadvertising, est une stratégie publicitaire exacerbant les réactions affectives des récepteurs de publicités, pouvant aller jusqu'à entrâner des émotions violentes. Une des entreprises les plus célèbres usant de ce type de processus de persuasion est Benetton, avec ses campagnes percutantes grâce à sa collaboration avec le concepteur O. Toscani (Tantet, 1992). 
[De nombreuses campagnes actuelles] sont en parfaite adéquation avec le public décalé qu'elles visent, habitué à porter un regard détaché sur les codes publicitaires. En plus de masquer leur visée persuasive première, l'ironie permet aux annonceurs de séduire leur cœur de cible sans avoir à assumer un discours aujourd'hui politiquement incorrect, mais néanmoins encore d'actualité. Elle a en outre pour avantage de rejeter sur les récepteurs la responsabilité d'une interprétation inconvenante (2013).

Toutes ces raisons justifient donc le succès des affiches de 2011 où l'ironie découlant de l'association des images avec les accroches se révèle être l'élément qui a le plus séduit les personnes sondées. Le choc des images n'est pas étranger à la réussite de cette campagne puisque la transgression ou la violence des visuels se révèlent aussi être un moyen efficace de sensibilisation dans la mesure où elles rompent la routine publicitaire, surprenant ainsi le spectateur (Ortega, 1997, 2004). R. Eguizábal va encore plus loin en indiquant que "hay algunos publicitarios que han identificado la publicidad con el ejercicio de la provocación, es decir, con el desarrollo de una comunicación en el límite de lo social, de lo político o incluso de lo legalmente aceptable ${ }^{15 "}$ (2007: 63). Dans les affiches dénonçant les méfaits des excès de l'agriculture industrielle, il est clair que l'association cherche à inquiéter les individus afin qu'ils prennent part au combat écologique. Il est ici question d'une technique publicitaire qui ne cesse de porter ses fruits: des résultats de recherche ont dès lors témoigné qu'avoir recours à la peur dans les campagnes de santé publique ou sécurité routière engendre une stimulation émotionnelle chez les destinataires qui les incite à changer leurs comportements à risque pour des attitudes plus saines ou sûres (Janezic, 2015). La deuxième série d'affiches a, quant à elle, captivé son public grâce à ses slogans encourageants. Adoptant une toute autre stratégie discursive pour sa campagne "Vivre dans un monde vivable", FNE a ici convaincu en transmettant des messages enthousiastes or "sin duda los anuncios que convencen conectan emocionalmente con la audiencia. Y no sólo convencen, sino que gustan y emocionan ${ }^{16}$, (Los Ángeles, 2005: 21). Néanmoins, les individus ayant participé au sondage ont désapprouvé la longueur des formules dans les deux campagnes. Rappelons ici la définition de slogan: "phrase publicitaire concise et originale, conçue en vue de bien inscrire dans l'esprit du public le nom d'un produit, d'une firme ${ }^{17}$ ", une description qui insiste sur le caractère bref de l'énoncé pour une meilleure mémorisation. M. Desnica (2016) dans son analyse des publicités pour la marque Schweppes remarquait de la même manière que leurs slogans trop extensifs amenuisaient leur compétitivité, la brièveté du texte publicitaire étant un atout non négligeable dans la captation du public.

15 Certains annonceurs ont identifié la publicité avec l'exercice de la provocation, c'est-à-dire, avec le développement d'une communication à la limite du socialement, politiquement ou même légalement correct (traduction personnelle).

16 Sans aucun doute les annonces qui convainquent communiquent émotionnellement avec l'audience. Et non seulement elles convainquent, mais elles plaisent et émeuvent (traduction personnelle).

17 < https://www.larousse.fr/dictionnaires/francais/slogan/73057> [04/06/2020]. 


\section{Conclusions}

Ce travail de recherche centré sur les publicités sociétales, et tout spécialement sur les deux campagnes de FNE datant de 2011 et 2016, nous a permis de repérer et d'analyser deux stratégies publicitaires distinctes pour transmettre une même idée, à savoir l'importance de la protection de l'environnement. L'emploi de l'ironie ou de slogans rassurants sont en effet deux techniques discursives employées en marketing, tant pour les campagnes sociales que pour les publicités commerciales. Leur incidence sur les individus est bien évidemment différente puisqu'elles engendrent deux types d'émotions clairement dissemblables. Ainsi, dans la campagne de 2011, le recours au facteur ironie n'occulte que légèrement la gravité du message et son impact sur les destinataires des affiches se voit intensifié par le choc psychologique que produit l'association images et slogans. Dans celle de 2016, les propos réconfortants et prometteurs d'un avenir salutaire ne choquent en rien les individus. Dans ce cas de figure, les concepteurs des affiches ont souhaité toucher la sensibilité des destinataires, mais surtout leur envie croissante d'un monde plus sain. Le sondage effectué, bien que méritant un échantillon plus vaste pour une étude plus complète, nous a apporté de premiers résultats éloquents quant à l'efficacité de ces deux campagnes. Il est clair que toutes deux semblent convaincre à part égale et font également l'objet de quelques critiques: dans la première, les blâmes portent sur la longueur des formules alors que dans la seconde est pointée du doigt la suavité des images. Il est finalement question de deux campagnes qui, comme tout objet publicitaire, amènent les consommateurs que nous sommes à développer des sentiments à connotation positive ou négative, qui finissent par nous convaincre de modifier nos comportements.

Il est manifeste que les questions environnementales préoccupent de plus en plus les populations occidentales, raison pour laquelle les publicités commerciales exploitent l'argument du naturel tandis que les campagnes sociétales à ce sujet se développent. Ces deux catégories publicitaires méritent donc des études de caractère pluridisciplinaire et constamment actualisées afin de comprendre au mieux leurs fonctionnement et efficacité. Dans le domaine de recherche qui nous concerne, de nombreuses interrogations demeurent quant aux effets des éléments sémio-linguistiques et discursifs des affiches publicitaires. Comme mentionné dans cet article, nous pourrions par exemple évaluer la teneur politique des messages diffusés par les associations non gouvernementales, recenser et identifier les discours fallacieux du greenwashing, ou encore développer notre analyse des recours à l'humour. En un mot, la publicité est un champ de recherche inépuisable nécessitant des études régulières et transversales. 
Anales de Filología Francesa, n. ${ }^{\circ} 28,2020$

NATURE ET PUblicité SOCIALE: DU SCÉNARIO CATASTROPHe À LA RECONQUÊTE DE...

\section{Références bibliographiques}

Abraham, Marine (2017). Le discours publicitaire destiné aux adolescents français: ambivalences linguistiques et sémiotiques et implications socioculturelles. Tesis Doctoral, Universidad de Murcia.

Becheur, Imène et Valette-Florence, Pierre. 2014. "L'usage des émotions négatives en communication de santé publique: étude des effets de la peur, la culpabilité et la honte" in $R e$ cherche et applications en marketing, Vol. 29, N4, 96-119. <https://halshs.archives-ouvertes. fr/halshs-01373948> [05/06/2020].

Bonhomme, Marc et Pahud, Stéphanie. 2013. "Un renouveau actuel de la rhétorique publicitaire?" in Semen, No36 [En ligne]. <https://journals.openedition.org/semen/9633> [07/06/2020]. DOI: https://doi.org/10.4000/semen.9633.

Cossette, Claude. 2001. La publicité, déchet culturel. Québec, Presses de l’Université de Laval.

Dictionnaire en ligne Larousse. <www.larousse.fr> [30/05/2020].

Desnica, Mirta. 2016. "Diffusion et déformation d'un slogan publicitaire mémorable dans la presse écrite: le cas de 'Nespresso. What else?"' in Travaux de Linguistique, No73/2, 69-83. $<$ https://www.cairn.info/revue-travaux-de-linguistique-2016-2-page-69.htm> [02/06/2020]. DOI: https://doi.org/10.3917/t1.073.0069.

Dorey, Françoise et Zollinger, Monique. 2000. "L'humour et le genre dans le discours publicitaire" in Décisions Marketing, No20, 57-65. <http://www.jstor.org/stable/40592745> [02/06/2020]. DOI: https://doi.org/10.7193/DM.020.57.65.

EguizÁbal, Raúl. 2007. Teoría de la publicidad. Madrid, Catedra.

EguizÁBAL, Raúl. 2008. "De la publicidad como actividad de producción simbólica” in

Hellín Ortuño, Pedro (éds.). El discurso publicitario contemporáneo. Su relación con lo social. Murcia, Diego Marín, 25-44.

France Nature Environnement. <https://www.fne.asso.fr/> [15/05/2020].

Garabuau-Moussaoui, Isabelle. 2011. "L'énergie est-elle un enjeu de pouvoir dans la famille?" in BARrey, Sandrine \& Emmanuel KESSOUS (éds.). Consommer et protéger l'environnement: opposition ou convergence? Paris, L'Harmattan, 67-88.

HÉrAult, Stéphanie. 2012. "Responsabilité sociale de l'entreprise et publicité" in RIMHE, Vol. 1, No 1, 7-18. <https://www.cairn.info/revue-rimhe-2012-1-page-7.htm> [25/05/2020]. DOI: https://doi.org/10.3917/rimhe.001.0007

JANEZIK, Isidora. 2015. "Empathie comme variable médiatrice de persuasion: le cas des publicité sociales antidiscrimination" in COMMposite, Vol. 18, No 1, 5-38. <http://www.commposite.org/index.php/revue/article/viewFile/213/173> [04/06/2020].

León, José Luis. 2001. Mitoanálisis de la publicidad. Barcelone, Ariel.

LóPez DíAz, Monteserrat. 2006. "Des 'humours' du discours publicitaire” in Chabrol, 
Claude (éds.). Questions de communication, No10, 119-134. <https://journals.openedition. org/questionsdecommunication/7693> [04/06/2020]. DOI: https://doi.org/10.4000/questionsdecommunication.7693.

Los Ángeles, Juan. 2005. “Aproximación al fenómeno de la publicidad. Los profesionales de la publicidad y la escritura publicitaria" in Romero, $\mathrm{M}^{\mathrm{a}}$ Victoria (éds.). Lenguaje publicitario, la seducción permanente. Barcelone, Ariel, 13-24.

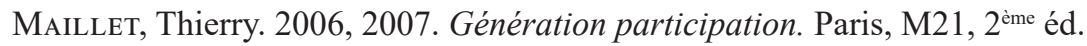

Martin, Stéphane (sous la direction de). 2017. Bilan 2017 Publicité \& Environnement. Paris, ARPP. <https://www.arpp.org/actualite/publicite-et-environnement-2017/> [20/05/2020].

Ortega, Enrique. 1997, 2004. La comunicación publicitaria. Madrid, Pirámide, $2^{\text {ème éd. }}$

PhILliPs, Barbara et McQuarrie, Edward. 2004. "Beyond visual metaphor: a new typology of visual rhetoric in advertising" in Marketing theory, $\mathrm{N}^{\circ} 4,113-136 .<$ http://mtq.sagepub. com/content/4/1-2/113.abstract $>$ [04/06/2020].

Soulages, Jean-Claude. 2006. "Les stratégies humoristiques dans le discours publicitaire"in Chabrol, Claude (éds.). Questions de communication, $\mathrm{N}^{\circ} 10,103-118$. $<$ https://journals.openedition.org/questionsdecommunication/7692> [04/06/2020]. DOI: https://doi.org/10.4000/ questionsdecommunication.7692.

TAntet, Marie. 1992. "La stratégie publicitaire de Benetton" in Communication et Langages, N $^{\circ}$ 94, 20-36. <https://www.persee.fr/doc/colan_0336-1500_1992_num_94_1_2392> [25/05/2020]. DOI : https://doi.org/10.3406/colan.1992̄.2392.

Ville de Paris. < https://www.paris.fr/pages/mesures-prises-en-cas-de-pic-de-pollution-2720> [31/05/2020].

Zhang, Yong. 1996. "Responses to Humorous Advertising: The Moderating Effect of Need for Cognition" in Journal of Adverting, Vol. 25, No 1, 15-32. <http://www.jstor.org/ stable/4188995> [02/06/2020]. DOI: https://doi.org/10.1080/00913367.1996.10673493

\section{Annexes}

\section{Annexe No1}

Transcription du sondage élaboré sur Google Formulaires en mai 2020 et disponible sur la page internet $<$ https://forms.gle/wJtdwCemTvvQHXoQ6>. 
Anales de Filología Francesa, n. ${ }^{\circ}$ 28, 2020

NATURE ET PUblicité SOCIALE: DU SCÉNARio CATASTROPHE À LA RECONQUÊTE DE...

Nature et publicité sociale : étude contrastive des campagnes de FNE.

Afin de mener à bien une étude contrastive entre les deux campagnes sociales lancées par France Nature Environnement (FNE) en 2011 et 2016, je vous remercie de bien vouloir compléter ce court questionnaire. II est bien évidemment anonyme mais si vous le souhaitez, je me ferai une joie de vous envoyer les résultats obtenus. Pour plus d'informations, contactez-moi par mail à l'adresse suivante : marine.abraham1@um.es. Vous trouverez ci-contre une vidéo regroupant les affiches des deux campagnes (sans son). Pour une meilleure compréhension des publicités, le visionnage de cette vidéo est recommandé. Merci d'avance.

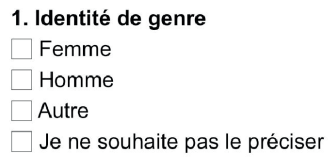

2. Âge

$\square$ Moins de 20 ans

Entre 20 et 40 ans

Entre 40 et 60 ans

Plus de 60 ans

$\checkmark$ Je ne souhaite pas le préciser

3. Quelle est l'influence des publicités commerciales sur vos achats ?
Excessive
Importante
Normale
Faible
Nulle

4. Généralement, où êtes-vous la/le plus à même de vous attarder sur des publicités ?

Dans l'espace urbain

Sur les pages internet et réseaux sociaux (Facebook, Instagram, Twitter, Youtube, etc.)

Dans la presse ou/et les magazines écrits

Par publipostage

Autres

5. Quelle est l'influence des publicités sociales sur vos comportements ?
Excessive
Importante
Normale
Faible
Nulle

6. Certaines campagnes de publicités sociales vous ont-elles tout particulièrement marqué(e) ?
Oui, beaucoup
Oui, quelques unes
Oui, mais peu
Non

\section{Si oui, lesquelles ?}

8. Connaissez-vous l'association France Nature Environnement (FNE) ?

Oui

Non 
Anales de Filología Francesa, n. ${ }^{\circ}$ 28, 2020

Marine Abraham

9. L'association FNE a lancé deux campagnes : la première contre l'excès de l'agriculture industrielle en 2011 et la seconde en 2016 intitulée "Vivre dans un monde vivable". Laquelle préférez-vous ?

$\square$ Campagne contre l'excès de l'agriculture industrielle (2011)

Campagne "Vivre dans un monde vivable"

Je n'ai pas de préférence

\section{Pourquoi?}

$\square$ La/Les thématique(s) abordée(s)

Le poids des images

La force des slogans

L'association slogan/image

Autres

11. Quel est, selon vous, le point positif de la campagne contre l'excès de l'agriculture industrielle (2011) ? Le choc des images

Le jeu des slogans

L'ironie émanant de l'association slogan et image

Le thème abordé : la campagne de 2011 se centre sur la lutte contre les excès de l'agriculture industrielle

La typographie (couleurs, formes, signes, polices, etc.)

Autres

II n'y a selon moi pas de points positifs

12. Quel est, selon vous, le point négatif de la campagne contre l'excès de l'agriculture industrielle (2011) ? $\square$ La violence des images

La longueur des slogans

L'ironie émanant de l'association slogan et image

Qu'il s'agisse d'une campagne à usage unique

La typographie (couleurs, formes, signes, polices, etc.)

L'absence de «nom de campagne »

$\neg$ Autres

II n'y a selon moi pas de points négatifs

13. Quel est, selon vous, le points positif de la campagne "Vivre dans un monde vivable" (2016) ?

$\square$ Les images idylliques en accord avec la nature

Les slogans porteurs d'espoir

La variété des thèmes abordés

Le nom de la campagne : "Vivre dans un monde vivable"

La typographie (couleurs, formes, signes, polices, etc.)

Autres

II n'y a selon moi pas de points positifs

14. Quel est, selon vous, le point négatif de la campagne "Vivre dans un monde vivable" (2016) ?

$\square$ La longueur des slogans

Les images lisses et bienveillantes

La variété des thèmes abordés

Le nom de la campagne : "Vivre dans un monde vivable"

La typographie (couleurs, formes, signes, polices, etc.)

Autres

II n'y a selon moi pas de points négatifs

15. Connaissiez-vous ces deux campagnes publicitaires ?

$\square$ Oui

Non 
